\title{
Surgery in a Patient with Liver Disease
}

\author{
Rakesh Rai $^{\star}$, Sanjay Nagral ${ }^{\star \star}$, Aabha Nagral ${ }^{\dagger}$ \\ *Department of HPB Surgery and Liver Transplantation, Fortis Hospital, Mulund, **Department of Surgical Gastroenterology, Jaslok Hospital, \\ and ${ }^{\dagger}$ Department of Gastroenterology, Jaslok Hospital, Mumbai, India
}

\begin{abstract}
Surgery is often needed in patients with concurrent liver disease. The multiple physiological roles of the liver places these patients at an increased risk of morbidity and mortality. Diseases necessitating surgery like gallstones and hernia are more common in patients with cirrhosis. Assessment of severity of liver dysfunction before surgery is important and the risk benefit of the procedure needs to be carefully assessed. The disease severity may vary from mild transaminase rise to decompensated cirrhosis. Surgery should be avoided if possible in the emergency setting, in the setting of acute and alcoholic hepatitis, in a patient of cirrhosis who is child class $\mathrm{C}$ or has a MELD score more than 15 or any patient with significant extrahepatic organ dysfunction. In this subset of patients, all possible means to manage these patients conservatively should be attempted. Modified Child-Pugh scores and model for end-stage liver disease (MELD) scores can predict mortality after surgery fairly reliably including nonhepatic abdominal surgery. Pre-operative optimization would include control of ascites, correction of electrolyte imbalance, improving renal dysfunction, cardiorespiratory assessment, and correction of coagulation. Tests of global hemostasis like thromboelastography and thrombin generation time may be more predictive of the risk of bleeding compared with the conventional tests of coagulation in patients with cirrhosis. Correction of international normalized ratio with fresh frozen plasma does not necessarily mean reduction of bleeding risk and may increase the risk of volume overload and lung injury. International normalized ratio liver may better reflect the coagulation status. Recombinant factor VIIa in patients with cirrhosis needing surgery needs further study. Intra-operatively, safe anesthetic agents like isoflurane and propofol with avoidance of hypotension are advised. In general, nonsteroidal anti-inflammatory drug (NSAIDs) and benzodiazepines should not be used. Intra-abdominal surgery in a patient with cirrhosis becomes more challenging in the presence of ascites, portal hypertension, and hepatomegaly. Uncontrolled hemorrhage due to coagulopathy and portal hypertension, sepsis, renal dysfunction, and worsening of liver failure contribute to the morbidity and mortality in these patients. Steps to reduce ascitic leaks and infections need to be taken. Any patient with cirrhosis undergoing major surgery should be referred to a specialist center with experience in managing liver disease. (J CLIN ExP НеPATOL 2012;2:238-246)
\end{abstract}

$\mathrm{T}$ The incidence of liver disease in India is on the rise. ${ }^{1}$ The number of surgical procedures performed including major operations is also increasing. Therefore, it is not uncommon for clinicians from various

Keywords: Anesthesia, Child-Pugh score, cirrhosis, coagulopathy, hepatic Received: 17.2.2012; Accepted: 15.5.2012; Available online: 20.8.2012 Address for correspondence: Aabha Nagral, Department of Gastroenterology, Jaslok Hospital, 7, Snehasagar, Prabhanagar, Prabhadevi, Mumbai 400025, India.

E-mail: aabhanagral@gmail.com

Abbreviations: ABG: arterial blood gas; ASA: American Society of Anesthesiologists; BNP: brain natriuretic peptide; COPD: chronic obstructive pulmonary disease; CTP: Child-Turcotte-Pugh; CVP: central venous pressure; FDP: fibrin degradation products; FFP: fresh frozen plasma; HPS: hepatopulmonary syndrome; ICG: indocyanine green; ICU: intensive care unit; INR: international normalized ratio; MELD: model for end-stage liver disease; NSAID: nonsteroidal anti-inflammatory drug; PICD: paracentesis-induced circulatory dysfunction; PT: prothrombin time; PTT: partial thromboplastin time; SBP: spontaneous bacterial peritonitis; TEG: thromboelastogram; TIPS: transjugular intrahepatic portosystemic shunt

http://dx.doi.org/10.1016/j.jceh.2012.05.003 specialties to be called upon to evaluate and reduce surgical risk in a patient with deranged liver profile.

In a patient being evaluated for surgery, liver disease needs to be ruled out if there is a high-risk history like regular, long-term alcohol consumption, blood transfusions, tattooing, IV drug abuse, and family history of liver disease. Clinical suspicion of liver disease should arise in any patient with palmar erythema, enlarged parotid glands, spider naevi, edema feet, gynecomastia and testicular atrophy in men or breast atrophy in women. The spectrum of liver dysfunction in a surgical patient could range from an incidental finding of mildly raised liver enzymes to severe dysfunction as seen in a patient with acute liver failure or decompensated cirrhosis. In severe dysfunction, potentially every organ system may be affected. ${ }^{2}$

In this review, we will discuss issues related to nonhepatic surgery in a patient with liver disease. Assessment of risk especially in surgical conditions commonly encountered in patients with cirrhosis, pre-operative optimization, intra-operative, and post-operative precautions are elaborated. 


\section{ASSESSING OPERATIVE RISK}

The risk of surgery in a patient with liver disease depends on various factors such as etiology and severity of the liver disease and the type of surgery. In a lifesaving procedure in an emergency, the assessment of risk is less relevant. In planned surgery, any degree of clinically evident liver dysfunction should be investigated thoroughly and optimization of the patient's medical condition and alternative approaches should be considered. The overall risk will also need to take the urgency of surgery and other coexisting medical illnesses into account.

\section{Acute Liver Disease}

A patient with acute hepatitis of any cause has a poor outcome following surgery. Friedman in an analysis of risk factors affecting surgical outcome in patients with liver disease identified acute hepatitis as an independent risk factor and suggested that it should be considered a contraindication for elective surgery. ${ }^{3}$ Major elective surgery for a patient with suspected acute hepatitis E, for example, should be deferred until the patient has recovered, barring some compelling reason for urgency such as a perforated viscus.

\section{Chronic Liver Disease}

In patients with chronic liver disease (CLD), outcomes correlate with the underlying hepatocellular function. There are two commonly used scoring systems for patients with liver dysfunction.

The Child-Turcotte-Pugh (CTP) ${ }^{4}$ has been used for decades to assess the severity of liver disease. The score can be calculated at the bedside and its familiarity has made the system withstand the test of time. Multiple studies have shown its independent prognostic value across varied clinical settings both medical and surgical. ${ }^{5}$ Patients with class C CTP score are not suitable candidates for major elective surgery. The main weakness of the CTP score stems from the subjective measurement of ascites and encephalopathy.

Malinchoc et al developed a model for end-stage liver disease (MELD) score to predict survival in patients who had undergone transjugular intrahepatic portosystemic shunt (TIPS). ${ }^{6}$ This statistical model predicted survival and identified those patients whose liver-related postTIPS mortality is 3 months or less. The MELD score is calculated from the objective values of serum bilirubin, serum creatinine, and international normalized ratio (INR). The MELD score has been evaluated in multiple studies and has proved to be effective in predicting surgical mortality in cirrhosis. ${ }^{7}$ Befeler et $\mathrm{al}^{8}$ performed a retrospective study of 53 patients with cirrhosis undergoing abdominal surgery, and concluded that the MELD provided a more accurate prediction of patient outcomes than the CTP scores. Although this study was small, it showed the superiority of MELD over CTP in predicting mortality in cirrhotic patients undergoing surgery. In a more recent study from the Mayo Clinic involving 772 patients with cirrhosis who underwent major nonhepatic surgery, multivariable analysis revealed that MELD score, American Society of Anesthesiologists (ASA) class, and age predicted mortality post-operatively. Thirty-day mortality was $5.7 \%$ in those with MELD score of less than 8 compared with more than $50 \%$ in those with MELD scores more than $20 .^{9}$ Further, Hanje and Patel have suggested that patients with MELD score of 10 or less can undergo elective surgery. In patients with MELD score of 10-15, elective surgery should be performed with caution and pre-operative optimization. On the other hand, in patients with MELD score of $>15$, surgery should be avoided. ${ }^{10}$

\section{Other Risk Factors for Morbidity and Mortality in Cirrhosis}

Ziser and Plevak ${ }^{11}$ reviewed 733 patients with cirrhosis who underwent surgical procedures (except liver transplantation) at the Mayo Clinic over an 11-year period. The mortality rate within 30 days of surgery was $11.6 \%$. Long-term follow-up showed that most deaths occurred within the first few months after surgery, when many patients succumbed to pneumonia or renal insufficiency. Table 1 lists the factors that were found by multivariate analysis to be independently predictive of peri-operative complications and of post-operative mortality. Though this study did not look at MELD scores as it was published prior to the popularity of the MELD scoring system, it

Table 1 Factors independently predictive of complications and mortality in patients with cirrhosis undergoing surgery. ${ }^{11}$

\begin{tabular}{ll}
\hline Predictors of complications & \multicolumn{1}{c}{ Predictors of mortality } \\
\hline Child-Pugh class B or C & Male gender \\
\hline Ascites & Child-Pugh class B or C \\
\hline Etiology of cirrhosis other than PBC & Ascites \\
\hline Elevated creatinine & $\begin{array}{l}\text { Etiology of cirrhosis other than } \\
\text { PBC }\end{array}$ \\
\hline Pre-operative infection & Pre-operative infection \\
\hline COPD & ASA physical status 4-5 \\
\hline Pre-operative upper GI bleeding & Respiratory surgery \\
Invasiveness of surgical procedure & \\
\hline Intra-operative hypotension & \\
ASA physical status 4-5 & \\
\hline
\end{tabular}

The cumulative effect of these risk factors increased the probability of developing a peri-operative complication is as follows:

- $9.3 \%$ risk of complications with 1 risk factor.

- $14.5 \%$ risk with 2 factors.

- $33.5 \%$ risk with 3 factors.

- $63 \%$ risk with 4 or 5 factors.

- $73.3 \%$ risk with 6 factors.

- $100 \%$ risk with 7 or 8 factors.

ASA: American Society of Anesthesiologists; COPD: chronic obstructive pulmonary disease; GI: gastrointestinal; PBC: primary biliary cirrhosis. 
quantified the risk of surgical procedure with several other parameters.

\section{Specific Disease-related Issues Alcohol}

Abstinence prior to elective surgery in those with history of excessive alcohol consumption needs to be advised to avoid alcohol withdrawal in the peri-operative period, even in the absence of significant liver disease. These patients also have a higher risk of hepatotoxicity from drugs such as paracetamol.

\section{Autoimmune Liver Disease}

Patients with autoimmune liver disease are often on steroids or have been on steroids in the past. This fact needs to be borne in mind with an increase in the steroid dose to cover the 'stressful' peri-operative period.

\section{Wilson Disease}

D-Penicillamine is the chelating agent used for treatment of Wilson disease and it interferes with wound healing. In elective surgery, the dose of the drug needs to be reduced pre-operatively and the reduced dose continued for a few weeks after the surgery until the wound has healed completely.

\section{Risk Assessment in Commonly Performed Operations in Patients with Liver Disease \\ Gall Stones}

Patients with cirrhosis are at increased risk of developing gall stone disease compared with noncirrhotic population. The prevalence of gall stone disease in cirrhosis can be up to $29 \% .{ }^{12}$ The odds ratio for peri-operative mortality in patients with liver disease who undergo cholecystectomy is 8.47. ${ }^{13}$ Perkins et al confirmed that a MELD score greater to or equal to 8 predicts an increased risk of post-operative complications in open cholecystectomy. ${ }^{14}$ In a patient with compensated cirrhosis and no significant portal hypertension, laparoscopic cholecystectomy can be safely performed. ${ }^{13}$ Forty-four studies including a meta-analysis of three randomized controlled studies comparing open with laparoscopic cholecystectomy were analyzed. Laparoscopic cholecystectomy was associated with a lower operative time, reduced complication rates, and a shorter hospital stay. ${ }^{15}$ In a patient with decompensated cirrhosis, cholecystectomy should be avoided and if there is a dire need for intervention, a cholecystostomy may be performed.

It is not unusual to diagnose cirrhosis for the first time when a laparoscope is introduced for the removal of gallstones. The procedure is either abandoned or completed with increased risk of post-operative complications. Ideally, this situation should not arise and the cirrhosis should be picked up on blood tests or ultrasound imaging done pre-operatively for the gallstones. Marginally low serum albumin and platelet counts on blood tests and presence of an enlarged caudate lobe or a dilated portal vein or an enlarged spleen on ultrasound should alert the surgeon to the likely presence of a CLD.

\section{Herniae}

Incidence of umbilical and inguinal hernias is higher in patients with cirrhosis due to increased intraabdominal pressure secondary to ascites. Umbilical hernia can get complicated and lead to leakage, ulceration, rupture, and incarceration. These complications can lead to high mortality after emergency surgery. Therefore, an umbilical hernia should be repaired electively in cirrhotics before they develop complications. However, the control of ascites before and/or after repair is necessary. An attempt should be made to achieve this with diuretics and paracentesis. Portal decompression by TIPS if feasible allows better control of ascites. It has been observed that with a sudden resolution of ascites, the chances of obstruction of an umbilical hernia are higher as the sac which was preferentially occupied by the ascitic fluid can now accommodate the bowel. Therefore, the repair should be carried out soon after the TIPS. These patients should be managed in a tertiary center where facilities and experience to manage these patients are available. $^{16}$

Inguinal hernia on the other hand has a lower risk of complications and a higher risk of recurrence after surgery. Hence, elective surgical repair of an inguinal hernia is not recommended in patients with cirrhosis.

\section{Cellulitis}

Cirrhosis leads to immunodeficiency and these patients are at increased risk of infections. ${ }^{17}$ Besides, trauma to the edematous lower limb predisposes to infections and prevents early healing compared with those without edema. Although spontaneous bacterial peritonitis (SBP) is a common complication in a more recent series, cellulitis was detected in $19 \%$ of patients hospitalized with decompensated cirrhosis while SBP was presented in only $4 \%$ patients. Most common organisms causing bacterial skin infection in noncirrhotic population are gram-positive pathogens (mainly group A streptococci and Staphylococcus aureus), and infrequently caused due to gram-negative organisms. Skin infections in case of cirrhosis are most commonly due to gram-negative organisms. ${ }^{18,19}$ Thus, it is important to choose an antibiotic with gram-negative coverage in a cellulitis patient till culture results are available. Cellulitis should be treated early due to increased risk of septic complications in cirrhotics.

\section{Morbid Obesity Surgery}

Morbidly obese patients often have associated nonalcoholic steatohepatitis (NASH). About three quarters of 
bariatric surgery patients have liver steatosis, a quarter have fibrosis and $1-2 \%$ of patients will have cirrhosis as an unexpected finding on the table. ${ }^{20,21}$ In a recent study by Mosko et al, patients without cirrhosis undergoing bariatric procedures had lower mortality rates than those with compensated and decompensated cirrhosis. ${ }^{22}$

Thus, bariatric surgery in patients with cirrhosis could be carried out cautiously when cirrhosis is well compensated, but in patients with decompensated cirrhosis or with significant portal hypertension, bariatric surgery should be deferred. These patients are candidates for liver transplantation. Transjugular intrahepatic portosystemic shunt procedure has been described prior to the transplant followed by a bariatric procedure like laparoscopic banding to reduce the risk of portal hypertension-related bleeding.

\section{Cardiac Surgery}

Coronary artery bypass carries a higher risk in patients with cirrhosis. One of the largest series included 44 patients of which 7 patients died due to hepatic decompensation. ${ }^{23}$ A number of risk factors for hepatic decompensation following cardiac surgery were identified including CTP score, total time on cardiac bypass, and need of peri-operative pressor support. ${ }^{23}$ In a recent publication, Modi et al reviewed the data on patients with cirrhosis undergoing coronary artery bypass surgery and concluded that cardiac surgery could be offered to patients with Child-Pugh A and a low MELD score with mild increase in the risk of mortality. However, for patients with more advanced cirrhosis, the risk of mortality is un-acceptably high. Cardiopulmonary bypass may increase the risk of bleeding by inducing fibrinolysis, platelet dysfunction, and hypocalcemia. Revascularization without the use of cardiopulmonary bypass may be a lower risk but in these decompensated patients long-term survival is significantly poor and health status is compromised even well after cardiac surgery. ${ }^{24}$

\section{CONTRAINDICATIONS FOR SURGERY}

Friedman ${ }^{13}$ proposed the following list of contraindications to elective surgery in patients with liver disease:

\section{Acute viral hepatitis.}

2. Alcoholic hepatitis. In a retrospective series of patients with alcoholic hepatitis, the mortality rate was $58 \%$ among the 12 patients who underwent open liver biopsy, compared with $10 \%$ among the 39 who underwent percutaneous liver biopsy. ${ }^{25}$

3. Acute liver failure.

4. Acute renal failure.

5. Severe coagulopathy.

6. Hypoxemia.

7. Cardiomyopathy.

\section{PRE-OPERATIVE OPTIMIZATION}

All patients with clinically suspected liver disease should be assessed for evidence of decompensation in the form of jaundice, coagulopathy, ascites, renal dysfunction, electrolyte abnormalities, and encephalopathy. Factors known to exacerbate hepatic encephalopathy should be corrected though there is no evidence that prophylactic therapy can prevent encephalopathy after surgery. Patients with known varices should receive the appropriate prophylaxis and fluid overload should be avoided post-operatively.

The laboratory tests to assess these abnormalities also provide parameters necessary for calculation of the CTP and MELD scores. In patients with liver disease undergoing liver resection surgery, it is important to assess the hepatic reserve. There are multiple tests to assess the hepatic reserve ${ }^{26}$ but one which has been studied in detail is indocyanine green retention rate at $15 \mathrm{~min}$ (ICGR-15). ${ }^{27}$ These are less relevant in patients undergoing nonhepatic surgery. Computed tomography (CT) volumetry, assesses the residual volume and function of the remaining liver.

Along with the assessment of the severity of liver dysfunction, it is important in the pre-operative work-up to optimize the wide range of physiological derangements caused by declining liver function in patients with cirrhosis.

\section{Cardiovascular Function}

Irrespective of the etiology, patients with cirrhosis have a hyperkinetic and hyperdynamic circulation with tachycardia, low systemic blood pressure, and low left ventricular after load associated with splanchnic vasodilatation. Peri-operative vasoconstrictor infusion may be necessary to prevent further hypotension. In addition to alcohol, cirrhosis per se is associated with a cardiomyopathy known as cirrhotic cardiomyopathy which is characterized by blunted ventricular systolic and diastolic contractile responses to physical and pharmacological stress. ${ }^{28}$ The systolic dysfunction gets manifests during stressful procedures such as TIPS. It has also been implicated in renal dysfunction in the end-stage liver disease. The QT interval is prolonged in $50 \%$ of patients with cirrhosis and may manifest as a rhythmic disturbance which may be treated with beta-blockers. Also, drugs which prolong QT interval need to be used with caution. Routine cardiovascular testing is advisable in patients with cirrhosis with an electrocardiogram to detect a prolonged QT interval, which has been shown to significantly correlate with the severity of liver disease, elevated brain natriuretic peptide (BNP) level, and decreased survival. A 2D echo should also be done to identify systolic and diastolic dysfunction. Cirrhotic cardiomyopathy is best picked up by dobutamine stress echo. ${ }^{29}$ The prevalence of coronary artery disease is high in cirrhotic population where diabetes, alcohol abuse, and smoking are common. Pulmonary artery hypertension 
defined as the mean pulmonary artery pressure $>25 \mathrm{mmHg}$ is seen in $5 \%$ of patients with cirrhosis. ${ }^{30}$ It increases the risk of surgery, even in those with mild pulmonary hypertension and therefore the indication for surgery needs to be carefully evaluated.

\section{Respiratory Function}

Up to $40 \%$ of the patients with cirrhosis are hypoxic. Causes of hypoxia can be related to pleural effusion, tense ascites, or hepatopulmonary syndrome (HPS).$^{30}$ The cause of HPS is shunting of blood in pulmonary vascular bed due to dilatation of the pulmonary precapillary arterioles. In HPS, physical findings may reveal digital clubbing and cyanosis. During pre-operative work-up of a patient with cirrhosis, a measurement of arterial blood gas (ABG) should be done as a screening tool. $\mathrm{A} \mathrm{PaO}_{2}$ of $<80 \mathrm{mmHg}$ suggests significant hypoxemia and HPS should be ruled out by contrast echocardiography. ${ }^{31}$ There is no therapy which will improve oxygenation in case of HPS and only treatment is liver transplantation. As it carries a poor prognosis, any patient with HPS requiring surgery other than liver transplant should be explained the high-risk and if possible should be treated by nonsurgical interventions. ${ }^{32}$ Other causes of hypoxia-like pleural effusion and ascites need to be aggressively treated in the pre-operative period to optimize the patient's respiratory status before surgery. Various indices like the Goldman cardiac index have been used to assess the post-operative risk of cardiac and pulmonary complications. ${ }^{33}$

\section{Renal Dysfunction and Ascites}

In patients with cirrhosis, renal blood flow gets compromised due to splanchnic vasodilatation and glomerular filtration rate can be low in spite of a normal serum creatinine due to poor muscle mass. ${ }^{34}$ Pre-operative fasting and intra-operative fluid loss if not corrected appropriately can lead to functional renal failure in these patients. In patients with cirrhosis, there is a substantial risk for developing contrast-induced nephropathy; hence, any radiological examination which needs contrast should be avoided immediately before surgery. ${ }^{35}$ If contrast is necessary, then the patient needs to be well hydrated and low-volume non-ionic iso-osmolar agent should be used. ${ }^{36}$ Nonsteroidal anti-inflammatory drugs (NSAIDs) should be avoided as they may lead to renal dysfunction ${ }^{37}$ as well as increase the risk of variceal bleeding in cirrhosis. ${ }^{38}$ Among the antibiotics, aminoglycosides should also be avoided.

Ascites is associated with increased incidence of infections, renal failure, and worse long-term outcome. Patients with ascites present a significant challenge for abdominal surgery. The sudden loss of large volume ascitic fluid during surgery can lead to paracentesis-induced circulatory dysfunction (PICD), which is due to the activation of renin-angiotensin-aldosterone system. The incidence of
PICD is reduced by pre-emptive administration of intravenous albumin at $6-8 \mathrm{~g} / \mathrm{L}$ of ascites removed. ${ }^{39}$ Large volume ascites will benefit from pre-operative paracentesis, diuretic therapy, and stringent limitation of sodium intake to $2 \mathrm{~g}$ /day to minimize reaccumulation of the ascitic fluid. In a patient with refractory ascites planned for surgery, TIPS may be used to control the ascites if the patient does not have any contraindication for TIPS and has a MELD score $<18 .{ }^{40}$ This has also shown to decrease the portal hypertension to allow abdominal surgery to take place. ${ }^{41,42}$

\section{Hemostasis}

Coagulation is a complex interaction of procoagulation and anticoagulation pathways and the fibrinolytic system. ${ }^{43}$ Majority of plasma clotting factors and components of the fibrinolytic and anticoagulant pathways are synthesized in the liver.

Therefore, patients with liver disease have a risk of bleeding as well as thrombosis. Infections, endogenous heparinoids, and renal failure can also affect coagulation in patients with decompensated liver cirrhosis. ${ }^{44}$

Bleeding is also associated with thrombocytopenia secondary to hypersplenism, thrombopoietein deficiency, (myelosuppression due to hepatitis C, folate deficiency, and ethanol toxicity), autoantibodies and low-grade disseminated intravascular coagulation (DIC). ${ }^{45}$

Conventional coagulation tests include clotting time, PT, INR, and activated partial thromboplastin time. These measure only part of the process of thrombin generation. Further, it is not certain if INR reflects the true risk of bleeding in patient with liver disease. Since fibrinogen plays a role in the last step toward fibrin clot formation in the clotting cascade, serum fibrinogen and tests of fibrinolysis like clot lysis time may also be included.

Tests for global evaluation of coagulation-like thrombin generation time, and thromboelastography, ${ }^{46}$ and INR calibrated for cirrhosis (INR liver) ${ }^{47}$ may be more accurate in prediction of bleeding complications. Thrombin formation is globally measured by using a thrombin generation assay modified by the addition of thrombomodulin, and hence it assesses both plasma level of coagulation factors and also coagulation inhibitors in patients with liver disease. ${ }^{48}$ Thromboelastography measures clot formation, clot strength, and clot dissolution but does not measure vascular tone. It evaluates global hemostasis, though there have been conflicting reports on its reliability in different studies. ${ }^{49}$ It has been shown to be a significantly better predictor of post-operative bleeding than the coagulation profile. ${ }^{50}$ The INR liver is a modification of the standard INR where PT is calibrated using plasma from patients with cirrhosis instead of vitamin $\mathrm{K}$ antagonists and is thought to resolve variability of INR in these patients. However, these tests have not been prospectively evaluated in patients with 
liver disease. ${ }^{47,51}$ Recently, Tripodi et al described a simple laboratory method which evaluates protein $\mathrm{C}$ deficiency which could promote clotting in patients with cirrhosis. This test is a standardizable laboratory test which may determine the relative risk of clotting vs bleeding in patients with cirrhosis. ${ }^{52}$

A precise platelet count rather than 'adequacy' is necessary. Bleeding time would give an idea of platelet function though several more sensitive tests for platelet function have been described.

We would currently recommend a CBC, bleeding time, PT, PTT, and fibrinogen as minimum tests in all patients with cirrhosis needing surgery. However, efforts should be made to make thrombin generation assay and TEG available.

\section{Management of Peri-operative Bleeding in Patients with Cirrbosis}

Vitamin K: Although coagulopathy is usually due to poor hepatic function, cholestasis and concomitant poor nutrition or malabsorption due to gut edema could also lead to vitamin $\mathrm{K}$ deficiency. Vitamin $\mathrm{K}$ injection is given preferably IV in dose of $10 \mathrm{mg}$ for 3 days in patients with decompensated cirrhosis undergoing surgery. Oral vitamin $\mathrm{K}$ has no role.

Fresh frozen plasma and cryoprecipitate: The short halflife of factor VII in fresh frozen plasma (FFP) (3-5 h) necessitates administration of the FFP immediately before surgery and at frequent intervals during surgery with monitoring of coagulation parameters. ${ }^{53}$ Fresh frozen plasma contains all coagulation factors, inhibitors of coagulation, and fibrinolytic factors. The recommended dose of FFP is $10-15 \mathrm{~mL} / \mathrm{kg}$, although the response in patients with decompensated liver cirrhosis is unpredictable. Besides, correction of INR by FFP has not been shown to reduce the risk of bleeding. Also, FFP administration has been associated with significant problems including volume overload, exacerbation of portal hypertension, risk of infections, and risk of transfusion-related acute lung injury. ${ }^{54}$ Cryoprecipitate infusions are recommended for those with serum fibrinogen $<100 \mathrm{mg} / \mathrm{dL}$ in a dose of one bag of cryoprecipitate per $10 \mathrm{~kg}$ of body weight. This may be preferable to FFP as the volume required is much lower.

Platelet transfusions: Thrombocytopenia of $<50,000 / \mu \mathrm{L}$ for moderate risk and $100,000 / \mu \mathrm{L}$ for high-risk procedures should be corrected by platelet transfusion, which is ideally given at the start of surgery and not before surgery. ${ }^{55}$ Platelets are transfused in the dose of 1 unit per $10 \mathrm{~kg}$ of body weight, which means 5-8 units of whole blood-derived pooled donor platelets or 1 unit of single donor platelet for prophylaxis. Higher doses may be needed for patients with active bleeding. This is expected to raise the platelet count by approximately $30,000 / \mu \mathrm{L}$.
Desmopressin: Administration of 1-deamino-8-D-arginine vasopressin (DDAVP)-Desmopressin, an analog of anti-diuretic hormone vasopressin-has been used for hemostasis in conditions associated with platelet dysfunction like uremia. It shortens the bleeding time but not much benefit has been recorded in clinical trials. A recent study found intranasal desmopressin in a dose of $300 \mathrm{mcg}$ to be as effective as transfusion of blood products in achieving hemostasis in cirrhotic patients with moderate coagulopathy undergoing dental extraction. ${ }^{56}$

Antifibrinolytics: A hyperfibrinolytic state should be considered in the setting of delayed bleeding following a procedure or intractable oozing from a wound. Saliva and ascites have increased fibrinolytic properties, which is perhaps the rationale of using anti-fibrinolytics in patients with cirrhosis and intra-peritoneal or dental bleeding. Tranexamic acid has been used administered with a $10 \mathrm{mg} / \mathrm{kg}$ loading dose and repeated 3-4/day for a total of 2-8 days. ${ }^{57}$

Recombinant factor VIla: In severe bleeding during surgery, recombinant factor VIIa in the dose of $40 \mathrm{mcg} / \mathrm{kg}$ may be effective in the correction of coagulopathy and reducing blood product requirements. Its effect is transient and needs repeated dosing and is extremely expensive. Though, it corrects in vitro tests of coagulation, its benefit in patients with cirrhosis undergoing nonhepatic surgery has not been documented. ${ }^{58}$

Needless to mention, a major factor determining the extent of correction of coagulopathy during surgery should be the surgeon's assessment of the cause and severity of the on-going bleeding. Other measures such as maintaining low central venous pressure (CVP) and reducing the portal pressure during surgery are also helpful.

Hypercoagulable state may also occur in cirrhosis with risk of micro- and macrovascular thrombosis. ${ }^{59,60}$ Thrombotic complications in cirrhosis manifest usually as deep vein thrombosis, pulmonary embolism, and acute portal vein thrombosis. These can be carefully and safely treated with anticoagulants. ${ }^{51}$ Low-molecularweight heparin has shown to be of benefit in preventing portal vein thrombosis in cirrhosis in a recent randomized control study. ${ }^{61,62}$

In a nutshell, hemostasis in patients with decompensated liver disease is complex. Conventional tests for coagulation have a poor predictability for bleeding or thrombosis in these patients. Tests measuring global hemostasis including thromboelastogram (TEG) and thrombin generation time seem to be more predictive of clotting problems and need to be validated in future studies. Similarly, newer interventions to correct coagulation-like recombinant factor VIIa and antifibrinolytics need to be tested in a prospective manner. Until we have sufficient data, the conventional approach of partially correcting abnormal coagulation peri-operatively 
may be continued. However, this may not necessarily translate into clinically effective hemostasis.

\section{Malnutrition}

Patients with CLD have protein calorie malnutrition and impaired glucose tolerance. Also, they are often deficient in fat-soluble vitamins (cholestatic disorders) or trace elements and vitamins like zinc, magnesium, copper, folate, and $\mathrm{B}_{12}$. These deficiencies could delay wound healing and increase the risk of infections and complications.

About $80 \%$ of patients with cirrhosis suffer from malnutrition. ${ }^{63}$ Serum albumin, prealbumin, and triglyceride levels are helpful to objectively quantify the patient's nutritional status, in addition to observing physical signs of cachexia and wasting. Even in patient with child's class A, the prevalence of malnutrition can be as high as $25 \%{ }^{64}$ In the pre-operative period, every effort should be made to improve nutritional status before surgery. In January 2006, the European Society for Clinical Nutrition and Metabolism (ESPEN) issued specific guidelines on enteral nutrition in liver disease which can be easily applied in both inpatients and outpatients. ${ }^{65}$ The guidelines concluded that undernutrition adversely affects the prognosis in cirrhosis and simple bedside methods such as anthropometry or subjective global assessment (SGA) are considered adequate to identify patients at risk.

Several misconceptions related to diet in the setting of liver disease exist in India, aggravating the pre-existing malnutrition. A high protein diet up to $1-1.5 \mathrm{~g} / \mathrm{kg}$ body weight with sodium restriction to $2 \mathrm{~g}$ in those with ascites is used. Usually, patients with liver disease have loss of appetite; hence, feeding through a nasogastric tube should be considered. Branched-chain amino-acid-enriched formula may be used as a source of protein in those with hepatic encephalopathy. Placement of nasogastric or nasojejunal tube for feeding in the presence of varices does not increase the risk of bleeding.

\section{INTRA-OPERATIVE MANAGEMENT}

Pre-operative risk reduction strategies such as paracentesis, treatment of coagulopathy, hypovolemia, respiratory and renal dysfunction, and improving nutritional status should be employed and it is important for surgeons and anesthetists to be aware of the physiological changes caused by liver disease. Usually during any major surgery, arterial line and central venous line should be inserted to monitor intravascular volume, frequent blood sampling, and to administer vasoactive drugs if needed.

Because of the portal hypertension and associated coagulopathy, patients can have excessive bleeding. Besides meticulous surgical technique, the use of coagulation diathermy and energy sources like harmonic scalpel is preferred. Hemostatic continuous suturing should be preferred for suture lines. Monitoring of coagulopathy during prolonged procedures is necessary and a TEG in the operating room is a useful, quick adjunct. However, there is not much literature on the use of TEG in nonhepatic surgery in patients with cirrhosis.

\section{Anesthetic Management}

Patients with liver disease are more likely than patients without liver disease to have hepatic decompensation with the use of anesthesia. ${ }^{3}$

The decreased hepatic perfusion at baseline makes the cirrhotic liver more susceptible to hypoxemia and hypotension in the operating room. At baseline, hepatic arterial and venous perfusion of the cirrhotic liver may be decreased: portal blood flow is reduced as a result of portal hypertension, and arterial blood flow may be decreased because of impaired autoregulation. Anesthetic agents may reduce hepatic blood flow by $30-50 \%$, and agents such as isoflurane, desflurane, sevoflurane, and propofol, which cause less disturbance in hepatic arterial blood flow than other inhaled anesthetic agents, are preferred for patients with liver disease. ${ }^{66}$

Myocardial depression and vasodilatation are common to majority of anesthetic agents. These effects are of concern since many patients with advanced liver disease already have a hyperdynamic circulation because of peripheral vasodilatation. Spinal or epidural anesthetics may reduce the mean arterial pressure, which is of concern for similar reasons. Also, these routes are not preferred in those with coagulopathy or thrombocytopenia. Halothane, $20 \%$ of which is metabolized by the liver, is now uncommonly used and should be avoided in those with liver disease. ${ }^{67}$ In contrast, enflurane is only $4 \%$ metabolized by the liver. Isoflurane, desflurane, and sevoflurane have only minimal hepatic metabolism $(<0.2 \%)$, which makes them, along with nitrous oxide, the best anesthetic choices for patients with liver disease. In general, propofol is preferred to benzodiazepines as a narcotic. Among opioids, sufentanil and remifentanil are preferred ${ }^{68}$ and oxazepam is the preferred sedative in liver insufficiency.

The muscle relaxants, vecuronium and rocuronium, are metabolized exclusively by the liver and therefore avoided. ${ }^{69}$ Atracurium and cisatracurium are metabolized independent of the liver and are preferred in patients with liver disease. ${ }^{68}$

\section{POST-OPERATIVE MONITORING}

In patients with cirrhosis, liver failure is the most common cause of post-operative death. ${ }^{13}$ Depending upon the type of surgery and the severity of liver disease, it is important to monitor for expected complications and to pre-emptively take steps to prevent complications. In any patient with child $\mathrm{B}$ or $\mathrm{C}$ cirrhosis who undergoes surgery under general anesthesia, it is important for the patient to be monitored post-operatively in an ICU setting. These patients take 
longer time to completely recover from the effect of anesthetic agent and after extubation need close monitoring regarding their ventilation as they can get drowsy leading to hypoventilation. Urine output must be monitored carefully as intra-operative fluid shift can lead to poor renal perfusion which if not detected early and treated aggressively can lead to acute renal failure. In these patients, it is important to monitor the CVP, pulse, $\mathrm{BP}$, and oxygen saturation continuously.

Central venous pressure should be maintained between $8 \mathrm{~cm}$ and $12 \mathrm{~cm}$ of water, to maintain adequate organ perfusion without going into fluid overload. Possibly the most ominous peri-operative complication in a patient with liver disease is the onset of renal insufficiency, which may be precipitated by a number of factors, including nephrotoxic drugs and intra-operative hypotension. Renal insufficiency is usually a predictor of markedly reduced survival and a sign that hepatorenal syndrome may have developed. Any nephrotoxic drug should be avoided. Oral feeding should be started as soon as possible as that helps in preventing SBP. Post-operatively, liver, renal, and coagulation profile with blood sugars should be monitored closely to detect early liver failure or renal failure. Any deterioration in the liver or renal function could imply the presence of sepsis and there should be a low threshold for giving broad-spectrum antibiotics to these patients. As most of the opioids are metabolized by the liver, their dose should be reduced.

\section{CONCLUSIONS}

Surgery in a patient with liver disease carries specific and higher risks, which have been well identified and validated. Sound and planned pre-operative assessment, optimization, and appropriate peri-operative care can substantially reduce these risks. However, the necessity of extra investigations and increased supportive care adds to the financial burden which is relevant to the Indian situation. Hence, a balance between safety and resources needs to be maintained. A team approach to management where the impulse for surgical intervention is tempered by joint discussion across specialties is crucial in the decision-making process.

\section{CONFLICTS OF INTEREST}

All authors have none to declare.

\section{REFERENCES}

1. Amrapurkar D, Kamani P, Patel N, et al. Prevalence of nonalcoholic fatty liver disease: population based study. Ann Hepatol. 2007;6: 161-163.

2. Ozawa K, Aoyama H, Yasuda K, et al. Metabolic abnormalities associated with post operative organ failure. Arch Surg. 1983;118: 1245-1251.
3. Friedman LS. The risk of surgery in patients with liver disease. Hepatology. 1999;29:1617-1623.

4. Child CG, Turcotte JG. Surgery and portal hypertension. Major Probl Clin Surg. 1964;1:1-85.

5. Durand F, Valla D. Assessment of the prognosis of cirrhosis: ChildPugh versus MELD. J Hepatol. 2005;42(suppl 1):S100-S107.

6. Malinchoc M, Kamath PS, Gordon FD, Peine CJ, Rank J, ter Borg PC. A model to predict poor survival in patients undergoing transjugular intrahepatic portosystemic shunts. Hepatology. 2000;31:864871.

7. Bingener J, Cox D, Michalek J, Mejia A. Can the MELD score predict perioperative morbidity for patients with liver cirrhosis undergoing laparoscopic cholecystectomy? Am Surg. 2008;74:156-159.

8. Befeler AS, Palmer DE, Hoffman M, Longo W, Solomon H, Di Bisceglie AM. The safety of intra-abdominal surgery in patients with cirrhosis model for end-stage liver disease score is superior to Child-Turcotte-Pugh classification in predicting outcome. Arch Surg. 2005;140:650-654.

9. Teh SH, Nagorney DM, Stevens SR, et al. Risk factors for mortality after surgery in patients with cirrhosis. Gastroenterology. 2007;132:1261-1269.

10. Hanje AJ, Patel T. Preoperative evaluation of patients with liver disease. Nat Clin Pract Gastroenterol Hepatol. 2007;4:266-276.

11. Ziser A, Plevak DJ. Morbidity and mortality in cirrhotic patients undergoing anesthesia and surgery. Curr Opin Anaesthesiol. 2001;14:707-711.

12. Conte D, Fraquelli M, Fornari F, Lodi L, Bodini P, Buscarini L. Close relation between cirrhosis and gallstones: cross-sectional and longitudinal survey. Arch Intern Med. 1999;159:49-52.

13. Friedman LS. Surgery in the patient with liver disease. Trans Am Clin Climatol Assoc. 2010;121:192-205.

14. Perkins L, Jeffries M, Patel T. Utility of preoperative scores for predicting morbidity after cholecystectomy in patients with cirrhosis. Clin Gastroenterol Hepatol. 2004;2:1123-1128.

15. Laurence JM, Tran PD, Richardson AJ, Pleass HC, Lam VW. Laparoscopic or open cholecystectomy in cirrhosis: a systematic review of outcomes and meta-analysis of randomized trials. HPB (Oxford). 2012;14:153-161.

16. Triantos CK, Kehagias I, Nikolopoulou V, Burroughs AK. Surgical repair of umbilical hernias in cirrhosis with ascites. Am J Med Sci. 2011;341:222-226.

17. Caly WR, Strauss A. A prospective study of bacterial infections in patients with cirrhosis. J Hepatol. 1993;18:353-358.

18. Morris A. Cellulitis and erysipelas. Clin Evid. 2003;10:1878-1883.

19. Horowitz Y, Sperber AD, Almog Y, et al. Gram negative cellulitis complicating cirrhosis. Mayo Clin Proc. 2004;79:247-250.

20. Weingarten TN, Swain JM, Kendrick ML, et al. Nonalcoholic steatohepatitis (NASH) does not increase complications after laparoscopic bariatric surgery. Obes Surg. 2011;21:1714-1720.

21. Brolin RE, Bradley L, Taliwal RV. Unsuspected cirrhosis discovered during elective obesity operations. Arch Surg. 1998;133: 84-88.

22. Mosko JD, Nguyen GC. Increased perioperative mortality following bariatric surgery among patients with cirrhosis. Clin Gastroenterol Hepatol. 2011;9:897-901.

23. Suman A, Barnes DS, Zein NN, Levinthal GN, Connor JT, Carey WD. Predicting outcome after cardiac surgery in patients with cirrhosis: a comparison of Child-Pugh and MELD scores. Clin Gastroenterol Hepatol. 2004;2:719-723.

24. Modi A, Vohra HA, Barlow CW. Do patients with liver cirrhosis undergoing cardiac surgery have acceptable outcomes? Interact Cardiovasc Thorac Surg. 2010;11:630-634.

25. Greenwood SM, Leffler CT, Minkowitz S. The increased mortality rate of open liver biopsy in alcoholic hepatitis. Surg Gynecol Obstet. 1972;134:600-604. 
26. Gholson CF, Provenza JM, Bacon BR. Hepatologic considerations in patients with parenchymal liver disease undergoing surgery. Am J Gastroenterol. 1990;85:487-496.

27. Hemming AW, Scudamore $\mathrm{CH}$, Shackleton CR, Pudek M, Erb SR. Indocyanine green clearance as a predictor of successful hepatic resection in cirrhotic patients. Am J Surg. 1992;163:515-518.

28. Møller S, Henriksen JH. Cirrhotic cardiomyopathy. J Hepatol. 2010;53:179-190.

29. Wong F, Girgrah N, Graba J, Allidina Y, Liu P, Blendis L. The cardiac response to exercise in cirrhosis. Gut. 2001;49:268-275.

30. Krowka MJ. Hepatopulmonary syndrome and portopulmonary hypertension: implications for liver transplantation. Clin Chest Med. 2005;26:587-597.

31. Rodríguez-Roisin R, Krowka MJ, Hervé P, Fallon MB. Pulmonary-hepatic vascular disorders (PHD). Eur Respir J. 2004;24:861-880.

32. Schenk P, Schoniger-Hekele M, Fuhrmann V, et al. Prognostic significance of the hepatopulmonary syndrome in patients with cirrhosis. Gastroenterology. 2003;125:1042-1052.

33. Lawrence VA, Dhanda R, Hilsenbeck SG, Page CP. Risk of pulmonary complications after elective abdominal surgery. Chest. 1996;110:744-750.

34. Arroyo V, Jiménez W. Complications of cirrhosis: renal and circulatory dysfunction. Lights and shadows in an important clinical problem. J Hepatol. 2002;32:157-170.

35. Lodhia N, Kader M, Mayes T, Mantry P, Maliakkal B. Risk of contrast-induced nephropathy in hospitalized patients with cirrhosis. World J Gastroenterol. 2009;15:1459-1464.

36. Trivedi $\mathrm{H}$, Moore $\mathrm{H}, \mathrm{Nasr} \mathrm{S}$, et al. A randomized prospective trial to assess the role of saline hydration on the development of contrast nephrotoxicity. Nephron Clin Prac. 2003;93:29-34.

37. Wong F, Massie D, Hsu P, Dudley F. Indomethacin-induced renal dysfunction in patients with well-compensated cirrhosis. Gastroenterology. 1993;104:869-872.

38. De Lédinghen $\mathrm{V}$, Heresbach $\mathrm{D}$, Fourdan $\mathrm{O}$, et al. Anti-inflammatory drugs and variceal bleeding: a case-control study. Gut. 1999;44: 270-273.

39. Sola-Vera J, Minana J, Ricart E, et al. Randomized trial comparing albumin and saline in the prevention of paracentesis-induced circulatory dysfunction in cirrhotic patients with ascites. Hepatology. 2003;37:1147-1153.

40. Ferral H, Gamboa P, Postoak DW, et al. Survival after elective transjugular intrahepatic portosystemic shunt creation: prediction with model for end-stage liver disease score. Radiology. 2004;231: 231-236.

41. Azoulay D, Buabse F, Damiano I, et al. Neoadjuvant transjugular intrahepatic portosystemic shunt: a solution for extra hepatic abdominal operation in cirrhotic patients with severe portal hypertension. J Am Coll Surg. 2001;193:46-51.

42. Gil A, Martinez-Regueira F, Hernandez-Lizoain JL, et al. The role of transjugular intrahepatic portosystemic shunt prior to abdominal tumoral surgery in cirrhotic patients with portal hypertension. Eur J Surg Oncol. 2004;30:46-52.

43. Sinclair TS, Booth NA, Penman SM, Brunt PW, Mowat NA, Bennett NB. Protease inhibitors in liver disease. Scand J Gastroenterol. 1988;23:620-624.

44. Senzolo M, Cholongitas $\mathrm{E}$, Thalheimer $\mathrm{U}$, et al. Heparin-like effect in liver disease and liver transplantation. Clin Liver Dis. 2009;13:43-53.

45. Hugenholtz GGC, Porte RJ, Lisman T. The platelet and platelet function testing in liver disease. Clin Liver Dis. 2009;13:11-20.

46. Koh MB, Hunt BJ. The management of perioperative bleeding. Blood Rev. 2003;17:179-185.

47. Tripodi A, Chantarangkul V, Primignani M, et al. The international normalized ratio calibrated for cirrhosis (INR liver) normalizes prothrombin time results for model for end-stage liver disease calculation. Hepatology. 2007;46:520-527.
48. Tripodi A, Salerno F, Chantarangkul V, et al. Evidence of normal thrombin generation in cirrhosis despite abnormal conventional coagulation tests. Hepatology. 2005;41:553-558.

49. Bolliger D, Seeberger MD, Tanaka KA. Principles and practice of thromboelastography in clinical coagulation management and transfusion practice. Transfus Med Rev. 2012;26:1-13.

50. Spiess BD. Thromboelastography and cardiopulmonary bypass. Semin Thromb Hemost. 1995;21(suppl 4):27.

51. Caldwell SH, Hoffman M, Lisman T, et al. Coagulation disorder and heamostasis in liver disease: pathophysiology and critical assessment of current management. Hepatology. 2006;44:1039-1046.

52. Tripodi A, Primignani M, Lemma L, et al. Detection of the imbalance of procoagulant versus anticoagulant factors in cirrhosis by a simple laboratory method. Hepatology. 2010;52:249-255.

53. Bell CL, Jeyarajah DR. Management of the cirrhotic patient that needs surgery. Curr Treat Options Gastroenterol. 2005;8:473-480.

54. Mannucci PM. Abnormalities of heamostasis tests in chronic liver disease: clinically relevant?. In: Bavino IV, Franchis RD, eds. Portal Hypertension. Oxford, UK: Blackwell Publishing; 2006: 40-60.

55. Samama CM, Djoudi R, Lecompte T, Nathan-Denizot N, Schved JF. Agence Française de Sécurité Sanitaire des Produits de Santé expert group. Perioperative platelet transfusion: recommendations of the Agence Française de Sécurite Sanitaire des Produits de Santé (AFSSaPS) 2003. Can J Anaesth. 2005;52:30-37.

56. Stanca CM, Montazem AH, Lawal A, Zhang JX, Schiano TD. Intranasal desmopressin versus blood transfusion in cirrhotic patients with coagulopathy undergoing dental extraction: a randomized controlled trial. J Oral Maxillofac Surg. 2010;68:138-143.

57. Mannucci PM, Levi M. Prevention and treatment of major blood loss. N Engl J Med. 2007;356:2301-2311.

58. Franchini M, Montagnana M, Targher G, Zaffanello M, Lippi G. The use of recombinant factor VIla in liver diseases. Blood Coagul Fibrinolysis. 2008;19:341-348.

59. Lisman T, Caldwell SH, Burroughs AK, et al. Hemostasis and thrombosis in patients with liver disease: the ups and downs. $J$ Hepatol. 2010;53:362-371.

60. Northup PG, Sundaram V, Fallon MB, et al. Hypercoagulation and thrombophilia in liver disease. J Thromb Haemost. 2008;6:2-9.

61. Tsochatzis EA, Senzolo M, Germani G, Gatt A, Burroughs AK. Systemic review: portal vein thrombosis in cirrhosis. Aliment Pharmacol Ther. 2010;31:366-374.

62. Zecchini R, Ferrari A, Bemabucci V. Anticoagulant therapy is safe and effective in preventing portal vein thrombosis (PVT) in advanced cirrhotic patients: a prospective randomized controlled study. J Hepatol. 2010;52:S460.

63. Kondrup J. Nutrition in end stage liver disease. Best Pract Res Clin Gastroenterol. 2006;20:547-560.

64. Guglielmi F, Panella C, Buda A, et al. Nutritional state and energy balance in cirrhotic patients with or without hypermetabolism. Multicentre prospective study by the 'Nutritional Problems in Gastroenterology' Section of the Italian Society of Gastroenterology (SIGE). Dig Liver Dis. 2005;37:681-688.

65. Plauth M, Cabré E, Riggio O, Assis-Camilo M, Pirlich M, Kondrup J. ESPEN guidelines on enteral nutrition: liver disease. Clin Nutr. 2006;25:285-294.

66. Gelman S. General anesthesia and hepatic circulation. Can J Physiol Pharmacol. 1987;65:1762-1779.

67. Gut J. Molecular basis of halothane hepatitis. Arch Toxicol Suppl. 1998;20:3-17.

68. Hoetzel A, Ryan H, Schmidt R. Anesthetic considerations for the patient with liver disease. Curr Opin Anaesthesiol. 2012;25:340347.

69. Lebrault C, Berger JL, D'Hollander AA, Gomeni R, Henzel D, Duvaldestin P. Pharmacokinetics and pharmacodynamics of vecuronium in patients with cirrhosis. Anesthesiology. 1985;62:601-605. 